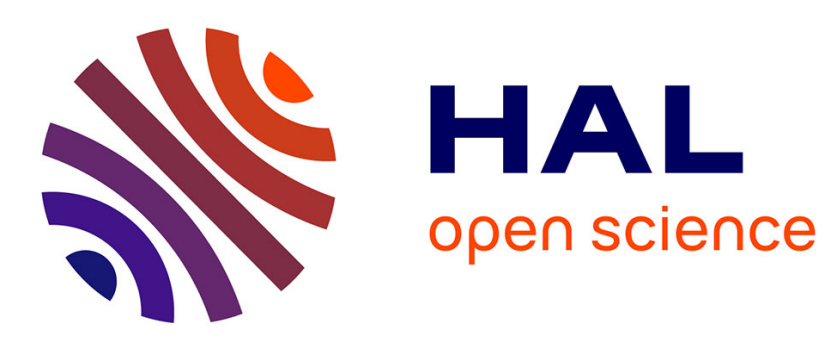

\title{
BRUIT AÉRODYNAMIQUE D'UNE TURBOMACHINE DE TYPE HÉLICO-CENTRIFUGE
}

\author{
H. Ameziane, S. Pauzin, J. Guilhot, O. Darnis
}

\section{To cite this version:}

H. Ameziane, S. Pauzin, J. Guilhot, O. Darnis. BRUIT AÉRODYNAMIQUE D'UNE TURBOMACHINE DE TYPE HÉLICO-CENTRIFUGE. Journal de Physique IV Proceedings, 1992, 02 (C1), pp.C1-605-C1-609. 10.1051/jp4:19921130 . jpa-00251087

\section{HAL Id: jpa-00251087 https://hal.science/jpa-00251087}

Submitted on 1 Jan 1992

HAL is a multi-disciplinary open access archive for the deposit and dissemination of scientific research documents, whether they are published or not. The documents may come from teaching and research institutions in France or abroad, or from public or private research centers.
L'archive ouverte pluridisciplinaire HAL, est destinée au dépôt et à la diffusion de documents scientifiques de niveau recherche, publiés ou non, émanant des établissements d'enseignement et de recherche français ou étrangers, des laboratoires publics ou privés. 


\title{
BRUIT AERODYNAMIQUE D'UNE TURBOMACHINE DE TYPE HELICO-CENTRIFUGE
}

\author{
H. AMEZIANE, S. PAUZIN*, J.P. GUILHOT ** et O. DARNIS \\ AREA/Technofan, Zac du Grand Noble, 10 place Marcel Dassaul, F-31702 Blagnac cedex, France \\ -AREA/CERT ONERA, 2 Av. Edouard Belin, F-31055 Toulouse cedex, France \\ - AREA/Université Paul Sabatier, LAMI (Laboratoire d'Acoustique de Métrologie el d'Instrumentation), \\ 38 rue des 36 Ponts, F-31062 Toulouse, France
}

\section{Résumé:}

L'étude presentée porte sur le calcul du bruit árodynamique d'une turbomachine (modèle de Lowson (Ref.1)). Le type hélico-centrifuge est plus particulièrement étudie. Les équipements, objets de nos investigations, sont des petites turbomachines qui tournent à vitesse elevé (environ $14000 \mathrm{tr} / \mathrm{mn}$ ), utilisées dans le domaine aéronautique et spatial. Une validation expérimentale et qualitative du modèle est réaliste en vue de réduire le bruit de raies.

\section{Abstract:}

This study concerns aerodynamic noise calculations of a mixod-flow turbomachine (Lowson model (Ref.1)). The equipment on wich we are working is small with high rotation speed (about $14000 \mathrm{rpm}$ ) and is used in aeronautics. An experimental validation is obtained by reducing the discrete frequency sound.

\section{Introduction:}

L'étude que nous présentons ici est la poursuite d'un travail rélisé au sein du laboratoire AREA sur le bruit aérodynamique rayonné par une roue de ventilateur hélico-centrifuge (Ref. 2).

Nous nous intéressons au bruit de raies et le formalisme choisi pour débuter l'étude est celui de Lowson. Nous exposons la formulation de la puissance acoustique rayonné par une roue sans distributeur amont et les difficultés auxquelles nous sommes confrontés, en particulier pour le calcul des harmoniques de charges. Nous présentons ensuite quelques modifications du modẻle, en vue de l'adapter plus efficacement à notre turbomachine.

Une étude parametrique nous permet de vérifier ce modzle, au moins d'un point de vue qualitatif. C'est ainsi que plusieurs roues ont été testées dans le but de réduire la puissance acoustique rayonnée.

Les mesures ont été faites à l'aspiration, en chambre sourde, selon la norme NF S31021 (Ref.3).

\section{1) Puissance sonore rayonnée oar une force ponctuelle fluctuante en mouvement -}

\subsection{Modèle de Loroson}

S'appuyant sur la theorie de Lighthill, Lowson exprime la pression sonore d'une force en mouvement. Après développement de la pression en série de Fourier, il établit l'expression de l'amplitude complexe de la pression acoustique du nième harmonique du bruit émis :

$$
P_{n}=\frac{i n B^{2} \omega}{2 \pi c_{0} r_{1}} \sum_{\lambda=-\infty}^{+\infty}(-i)^{n B-\lambda}\left(\frac{x}{r_{1}} F_{x}(\lambda)-\left(\frac{n B-\lambda}{n B M}\right) F_{y}(\lambda)\right) J_{n B-\lambda}\left(\frac{n B M y}{r_{1}}\right)
$$


Les deux hypothèses principales consistent, d'une part, à négliger l'effet de cascade, c'est à dire la directivite azimutale et, d'autre part, à ne pas détailler le champ de force s'exerçant sur l'aube, la corde étant petite devant la longueur d'onde (compacité).

Nous avons choisi la même loi empirique que Lowson pour exprimer les forces fluctuantes sur les aubages, qui traduit une décroissance exponentielle des harmoniques de charges. La décomposition en série de Fourier de la force fluctuante s'exprime par:

$$
\left(\begin{array}{l}
F_{x} \\
F_{y}
\end{array}\right)=\sum_{\lambda=-\infty}^{\lambda \rightarrow+\infty}\left(\begin{array}{l}
F_{x s} \\
F_{y s}
\end{array}\right) \lambda^{-h} \exp (-i \lambda \omega t)
$$

Un premier calcul fait apparaître quelques désaccords avec l'expérimentation (écart sur le niveau à partir du deuxième harmonique cf. Fig. 1).

D'une part, le côté arbitraire du paramètre $h$ n'est pas satisfaisant, d'autre part, pour un $h$ donné, on ne retrouve pas le spectre de raies mesure, en particulier pour les harmoniques d'ordre supérieur. Comptetenu de la difficulte d'obtenir des informations sur les harmoniques de charges par le calcul ou la mesure, le problème reste posé. Toutefois, l'étude des directivités en gisement et azimut peut, peutêtre, nous permettre de résoudre en partie ce problème, puisqu'elles nous renseignent sur l'importance des harmoniques de charges. Deux conséquences majeures, et que nous vérifierons expérimentalement, découlent de ce calcul, d'un point de vue qualitatif. La première est qu'une augmentation du nombre d'aubages de la roue pour des performances árauliques équivalentes réduit la puissance acoustique rayonnée. Il en va de même d'une diminution de l'écart entre la force axiale $F_{x s}$ et tangentielle $\mathrm{F}_{\mathrm{ys}}$ (augmentation de portance).

\subsection{Prise en compte de l'effort_radial}

Le ventilateur que nous étudions est de type hélico-centrifuge, c'est à dire que le fluide a une direction méridienne incliné par rapport à l'axe de rotation. Il apparaît donc intéressant d'introduire dans le calcul de Lowson une composante radiale des efforts stationnaires s'exerçant sur les aubages (Fig 3). On en déduit une nouvelle expression de la pression acoustique du bruit émis :

$$
P_{n}=\frac{i n B^{2} \omega}{2 \pi c_{0} r_{1} \lambda=\infty} \sum_{=\infty}^{+\infty}(-i)^{n B-\lambda}\left(\frac{x}{r_{1}} F_{x}(\lambda) \cos \left(a_{1}\right)-\left(\frac{n B-\lambda}{n B M}\right)\left(F_{y}(\lambda)+R_{e} F_{x}(\lambda) \frac{\sin \left(a_{1}\right)}{y}\right)\right) J_{n B-\lambda}\left(\frac{n B M y}{r_{1}}\right)
$$

Le calcul effectue avec cette nouvelle expression reste sensiblement identique au premier. L'effort radial est négligeable pour le calcul de la puissance acoustique rayonnée, d'où l'intérêt d'un ventilateur hélico-centrifuge. Résultat que nous retrouvons dans notre étude expérimentale.

\subsection{Calcul des forces sur les aubages. Point d'application de la résultante des forces.}

Notre calcul des forces suppose que le point d'application de la résultante est situé au rayon quadratique moyen $R_{e}$, calcule à partir des valeurs quadratiques moyennes des rayons en entré et en sortie d'aubage. Notre manque d'informations sur la distribution des pressions sur l'intrados et l'extrados de l'aubage nous empêche de vérifier cette hypothèse. Toutefois, les caractéristiques géométriques de notre profil nous permettent de choisir une corrélation NACA (Ref.4) pour laquelle nous disposons de résultats de mesure de pression et ceci pour plusieurs incidences. Nous présentons des résultats de calcul des forces et du point d'application de la résultante effectués à partir des distributions de pression mesurées.

\section{4- Directivité en gisement - Directivité azimutale}

Le modele de Lowson nous permet le calcul de la directivité en gisement en amont de la roue. Ce calcul est particulièrement intéressant puisqu'il peut éventuellement nous permeture de confirmer ou non l'importance de certains harmoniques de charges. Rappelons ici que le modele choisi ne prend pas en compte de directivité azimutale puisqu'il néglige le terme de phase azimutale. Il apparaît intéressant pour notre calcul d'introduire ce terme de phase qui nous permettra peut être de préciser davantage le rayonnement respectif de chaque harmonique de charge. Le calcul applique au fondamental du bruit de 
raies sera comparé à la mesure de directivité. Nous en déduirons un nouveau calcul de la puissance acoustique rayonnée par la roue.

\section{2) Etude expérimentale}

\section{1- Mesure}

Toutes les mesures de puissance acoustique sont effectuées en chambre sourde selon la norme française NF S31021. La surface de mesurage est un hémisphère d'un mètre de rayon qui s'appuie sur le plan d'entrée du ventilateur. Il y a neuf points de mesurage. Le niveau de puissance $\mathrm{L}_{w}$ est calculé à partir du niveau de pression moyen $\mathrm{L}_{\mathrm{pm}}$.

$\mathrm{L}_{\mathrm{w}}=\mathrm{L}_{\mathrm{pm}}+10 \log (2 \pi)$

La chaîne de mesure est constituée d'un analyseur et d'un microphone 1/4 pouce.

\section{1- Résultats}

L'étude expérimentale nous a conduit à réaliser plusieurs roues de type axial et hélico-centrifuge. Nous nous sommes intéressés à la comparaison des types, à l'effet de la variation du nombre d'aubes, des efforts s'exerçant sur les aubages, et plus généralement des caractéristiques géométriques des profils. A titre d'exemple, deux roues hélico-centrifuges ont été réalisées; l'une comportant neuf aubes, l'autre 23. Les vitesses de rotation sont identiques. A leur point de fonctionnement nominal (identique pour les deux roues), une atténuation de la puissance acoustique globale de $5 \mathrm{~dB}$ a été obtenue (Fig. 2 et 3). On observe effectivement une très nette diminution des niveaux de raies, qui s'accompagne d'une faible augmentation du bruit large bande. D'une part les efforts par pales sont plus faibles, d'autre part l'écart entre les efforts axial et tangentiel est réduit. Ce résultat est en parfait accord avec le modèle de Lowson.

L'ensemble des résultats est présenté en terme de puissance acoustique, et de puissance acoustique spécifique. Un récapitulatif des différents paramètres et leurs effets sur le bruit est effectué.

\section{Conclusion}

L'étude du bruit aérodynamique d'une turbomachine de type hélico-centrifuge se poursuit. Au niveau du calcul nous tenterons d'appréhender au mieux les efforts s'exerçant sur les aubages et ceci par des investigations supplémentaires menées sur les directivités en gisement et en azimut. Nous avons pu vérifier certaines orientations données par le modèle de Lowson et donc le valider en partie, d'un point de vue qualitatif. En effet, des réductions des niveaux de bruit des raies ont été obtenues par la réalisation de nouvelles roues dont les choix pour leur géométrie et leur calcul ont été guidés par le modèle.

\section{Princioales notations:}

B: nombre de pales de la rove

$\omega$ : vitesse angulaire de la roue

$c_{0}:$ célérité du son

$r_{1}$ : distance entre le centre de la roue et l'observateur fixe

M: nombre de Mach de rotation au rayon équivalent

$\mathrm{R}_{\mathrm{e}}$ : rayon équivalent, point d'application de la résultante des forces

$x, y$ : coordonnées du point d'application de la force résultante

$\mathrm{J}_{\mathrm{nB}-\lambda}$ : fonction de Bessel de première espèce d'ordre $\mathrm{nB}-\lambda$

$\mathrm{F}_{\mathrm{x}}, \mathrm{F}_{\mathrm{y}}:$ composante axiale et tangentielle de la force resultante instationnaire

$F_{x s}, F_{y s}$ : composante axiale et tangentielle de la force résultante stationnaire

$\lambda$ : rang de l'harmonique de charge

$a_{1}$ : angle du filet moyen avec l'axe du ventilateur 


\section{Références:}

(1) M.V. LOWSON, "Theoretical analysis of compressor noise"; J.A.S.A. vol 47 p371-385 (1970)

(2) H.AMEZIANE, S.PAUZIN, J.P.GUILHOT, O.DARNIS, D.BIRON, "Mesure et prédiction du bruit d'une turbomachine de type hélico-centrifuge"; Communication au 12ème colloque d'aéroacoustique et d'hydroacoustique à l'ONERA Châtillon avril 1991; à paraître dans le journal de la SFA (février 1992)

(3) Norme NF S31021, "Mesurage en plate-forme du bruit émis par les ventilateurs à enveloppe" (4)L.J.HERRIG, J.C.EMERY, J.R.ERWIN, "Systematic two-dimensional cascade tests of NACA 65 series compressor blades at low speeds"; NACA technical note 3916. 


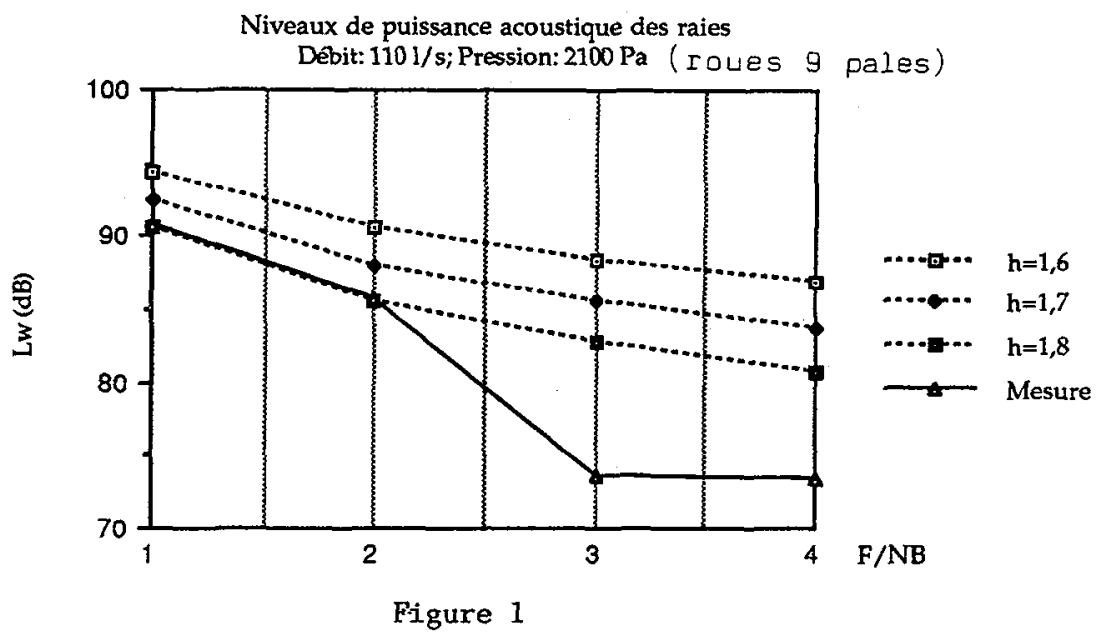

Spectre de puissance acoustique (roue 9 pales)

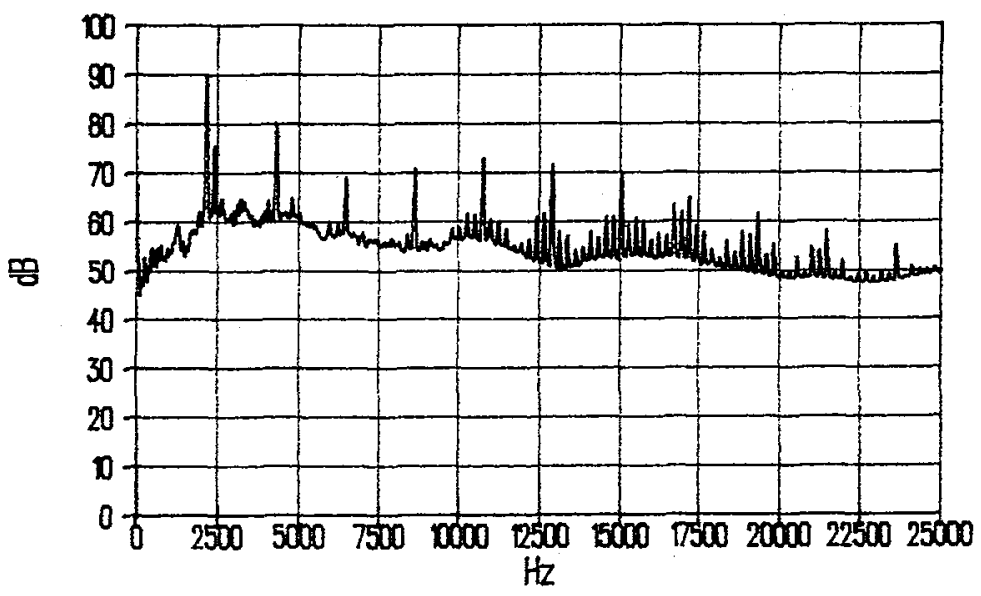

Figure 2

Spectre de puissance acoustique [roue 23 pales]



Figure 3 